\title{
Measurement of small photodestruction rates of cold, charged biomolecules in an ion trap
}

\author{
D. Offenberg, Ch. Wellers, C. B. Zhang, B. Roth, and S. \\ Schiller \\ Institut für Experimentalphysik, Heinrich-Heine-Universität Düsseldorf, 40225 \\ Düsseldorf, Germany
}

\begin{abstract}
In this work, we demonstrate quantitative measurements of photodestruction rates of translationally cold, charged biomolecules. The long-term stable storage of the molecular ions in an ion trap at ultra-high vacuum conditions allows measurement of small rates and verification that rates are linear in photodestruction laser intensity. Measurements were performed on singly protonated molecules of the organic compound glycyrrhetinic acid $\left(\mathrm{C}_{30} \mathrm{H}_{46} \mathrm{O}_{4}\right)$, dissociated by a continuous-wave UV laser $(266 \mathrm{~nm})$ using different intensities. The molecules were sympathetically cooled by simultaneously trapped laser-cooled barium ions to translational temperatures of below $150 \mathrm{mK}$. Destruction rates of less than $0.05 \mathrm{~s}^{-1}$ and a cross section of $(1.1 \pm 0.1) \cdot 10^{-17}$ $\mathrm{cm}^{2}$ have been determined. An extension to tunable UV laser sources would permit high-resolution dissociation spectroscopic studies on a wide variety of cold complex molecules.
\end{abstract}


Measurement of small photodestruction rates of cold, charged biomolecules in an ion trap 2

\section{Introduction}

Photodissociation spectroscopy is a common tool to gain information on structures of biomolecules [1], to distinguish between different isomers [2] or to study the energetics and pathways of fragmentations [3]. The investigation of these processes in the gas phase provides advantageous conditions. Here, one can guarantee, by mass selection prior to spectroscopy, the sample does not contain dimers, aggregates or chemically modified molecules. In the low-pressure environment of the gas phase, the collision rate can be strongly reduced. This enables the study of the above processes with little influence from intermolecular interactions. The interpretation of the results by quantum chemical calculations will therefore generally be facilitated [4].

Trapped-ion photodissociation offers additional advantages. The long storage times in ion traps can extend the time scale of observable dissociation processes, such as blackbody infrared radiative dissociation (BIRD) [5] or the unimolecular dissociation of large biomolecules at low energies [6, 7], to rates smaller than $1 \mathrm{~s}^{-1}$ which cannot be obtained using other, non-trapping approaches [8, 9, 10]. Additionally, due to the strong spatial localization of the trapped ions, even single molecules can be studied [11 resulting in yet simplified environmental conditions. By cooling the trapped molecules, spectral congestion and inhomogeneous line broadening can be reduced and photodissociation spectra of high resolution can be obtained. A conventional way of cooling both internal and external degrees of freedom of molecular ions in traps is using cryogenic buffer gases [12, 13] leading to translational and internal temperatures of a few Kelvin, but no strong spatial confinement. A combination of sympathetic translational cooling via laser cooled atomic ions [14, 15] and internal cooling might be feasible via radiative cooling in a cryogenically cooled ion trap [16]. This promises to reach both much lower temperatures than with the conventional buffer gas method and simultaneously a stronger spatial confinement.

Few quantitative determinations of photodestruction rates have been performed. One of the earliest was the measurement of the wavelength-dependent photodestruction of $\mathrm{CH}_{4}^{+}$in a Paul trap, where cross sections in the order of $1 \cdot 10^{-19} \mathrm{~cm}^{2}$ were determined [17, 18]. Here, we demonstrate a quantitative measurement of the photodestruction rate of a large polyatomic molecular ion species. The measurement of the cross section was performed as a function of laser intensity, allowing to verify that only one-photon processes contribute.

We employ an apparatus that provides protonated molecular ions with masses up to 12400 Da produced by electrospray ionization (ESI) at sub-Kelvin translational temperatures [14, 15]. For this purpose, the molecular ions are stored in a linear quadrupole trap and are sympathetically cooled by simultaneously trapped laser-cooled

${ }^{138} \mathrm{Ba}^{+}$ions via their mutual Coulomb interaction. The organic compound glycyrrhetinic acid (mass $471 \mathrm{Da}$ ) is used as a test molecule as it is chemically stable, inexpensive and available at a reliably high ESI ion flux. A low-power, fixed-frequency continuous-wave (cw) UV laser at $266 \mathrm{~nm}$ was used to demonstrate two different methods for determining 
photodestruction rates of cold molecules. The first method is based on a continuous monitoring of the decay of the parent ion number during the dissociation. In the second method, the destruction process is characterized by extracting and counting the ions remaining after different UV exposure durations.

\section{Experimental setup and basic principles}

Our experimental setup [14, 15] is shown schematically in figure 1. It consists of an ESI ion source [19] for the production of singly or multiply charged, gas-phase molecular ions with a mass-to-charge ratio $m / z$ ( $z$ is the number of elementary charges) of up to $2000 \mathrm{Da}$, a quadrupole mass filter for the selection of specific molecular species, a $\mathrm{RF}$ octopole ion guide to transfer the selected molecular ions from the medium vacuum region of the ESI device to an ultra-high vacuum (UHV) chamber with a typical residual gas pressure of $<10^{-9} \mathrm{mbar}$, and a linear quadrupole trap in this UHV chamber to store the molecular ions for sympathetic cooling and the intended experiments.

The preparation of the laser-cooled ${ }^{138} \mathrm{Ba}^{+}$ion ensembles used for the sympathetic cooling of the molecular ions as well as the required laser setup (a $493 \mathrm{~nm}$ cooling and a $650 \mathrm{~nm}$ repumper laser) have been described in [20. Under appropriate lasercooling conditions, the barium ions arrange in ordered structures, so-called Coulomb crystals, that can be imaged with an intensified CCD camera (see figures 2 (b) and (c)). The images contain information on numbers and temperatures of both the fluorescing ${ }^{138} \mathrm{Ba}^{+}$ions and indirectly of the invisible sympathetically cooled molecular ions. Using molecular dynamics (MD) simulations, these data can be derived from structural deformations and blurring of the barium ion Coulomb crystals [21]. Further components for the detection of the trapped ions are an ion detector to count massselectively extracted ions and a radially mounted electrode for the excitation of species-

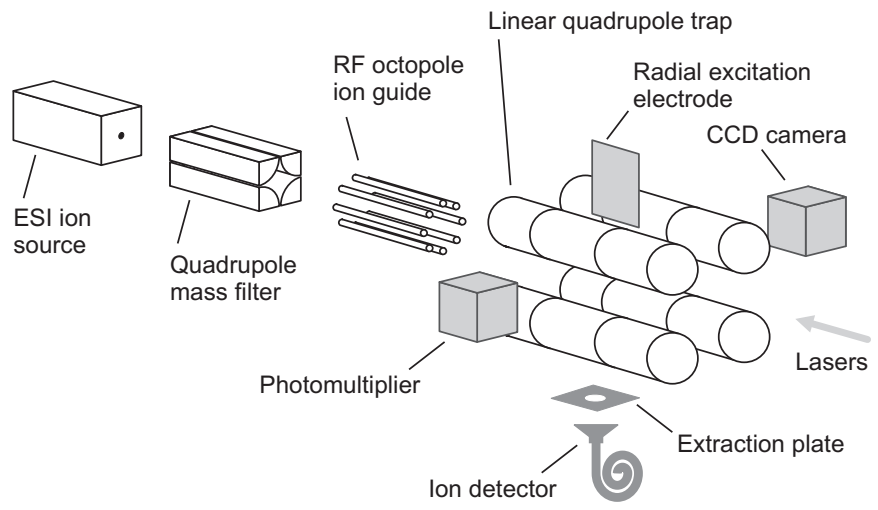

Figure 1. Schematic overview of the experimental setup. Protonated molecular ions from an ESI ion source are selected by a quadrupole mass filter and transferred via a RF octopole ion guide to a linear quadrupole trap in a UHV chamber. The trapped ions can be electronically excited by a radially mounted electrode and counted by an ion detector below the trap. The laser-cooled ${ }^{138} \mathrm{Ba}^{+}$ions are imaged with a CCD camera and their fluorescence is detected by a photomultiplier. 
specific oscillation modes. A resonant electric excitation causes a drop of the barium ions' fluorescence that can be measured with a photomultiplier.

Ensembles of cold $(<1 \mathrm{~K})$, singly protonated glycyrrhetinic acid molecules $\left(\mathrm{GAH}^{+}\right)$ produced by ESI are routinely prepared within less than $1 \mathrm{~min}$ by the following procedure 14, 15]. Here, with a glycyrrhetinic acid (Sigma-Aldrich) solution of $10^{-5} \mathrm{M}$ concentration in 1:1 acetonitrile:water with $5 \cdot 10^{-3} \%$ formic acid added for protonation, a flux of several ten thousand $\mathrm{GAH}^{+}$ions per second leaving the octopole ion guide is achieved. This flux can be switched on and off via the octopole RF voltage. For trapping the molecular ions, helium buffer gas at room temperature is injected into the trap chamber to reduce the ions' kinetic energy. The number of trapped ions depends linearly on the buffer gas pressure and the duration of the ion flux. For typical pressures of $1-10 \cdot 10^{-5}$ mbar several hundred molecular ions are trapped within a few seconds. After removal of the buffer gas, molecular fragment ions generated during loading are removed from the trap by applying an additional AC voltage frequency scan to the trap electrodes in order to excite the unwanted ions' specific motional resonances so strongly that these ions are ejected from the trap. Then, barium ions from an evaporator oven are loaded into the trap and cooled by the two cooling laser beams propagating along the trap axis. After two more frequency scans that remove impurity ions (such as $\mathrm{CO}_{2}^{+}$ and $\mathrm{BaO}^{+}$) generated during loading the barium, the preparation of a cold $\mathrm{Ba}^{+} / \mathrm{GAH}^{+}$ ion crystal is completed.

Figure 2 shows experimental and simulated CCD images of such an ion crystal revealing the spatial configuration of the different ion species. In a quadrupole trap, ions of different charge-to-mass ratios $Q / m$ arrange radially separated according to their effective radial trap potentials

$$
\Phi(r) \propto \frac{Q}{m} r^{2}
$$

and the interspecies repulsion $\sim Q_{1} Q_{2}$ [15]. For prolate crystals, this radial separation scales as

$$
\frac{r_{1}}{r_{2}} \simeq \sqrt{\frac{m_{1} / Q_{1}}{m_{2} / Q_{2}}}
$$

with the outer radius $r_{1}$ of the lower mass-to-charge ratio $m_{1} / Q_{1}$ subensemble and the inner radius $r_{2}$ of the higher mass-to-charge ratio $m_{2} / Q_{2}$ subensemble [22]. Thus, the heavier $\mathrm{GAH}^{+}$ions arrange around the barium ions and form a sheath which radially squeezes and axially prolongs the barium ion subensemble as shown in figure 2, From this structural deformation the number of trapped $\mathrm{GAH}^{+}$ions can be derived. Therefore, two experimental images are recorded - one with the molecular ions present and one after the molecular ions have been removed. In the case shown in figure 2 , the $\mathrm{GAH}^{+}$ ions prolong the barium ion subensemble by $252 \mu \mathrm{m}$ and radially squeeze it by $25 \mu \mathrm{m}$. According to MD simulations this can be explained by the presence of $1250 \pm 50 \mathrm{GAH}^{+}$ ions. The barium ion subensemble itself consists of $240 \pm 10$ laser-cooled ${ }^{138} \mathrm{Ba}^{+}$ions 
Measurement of small photodestruction rates of cold, charged biomolecules in an ion trap5

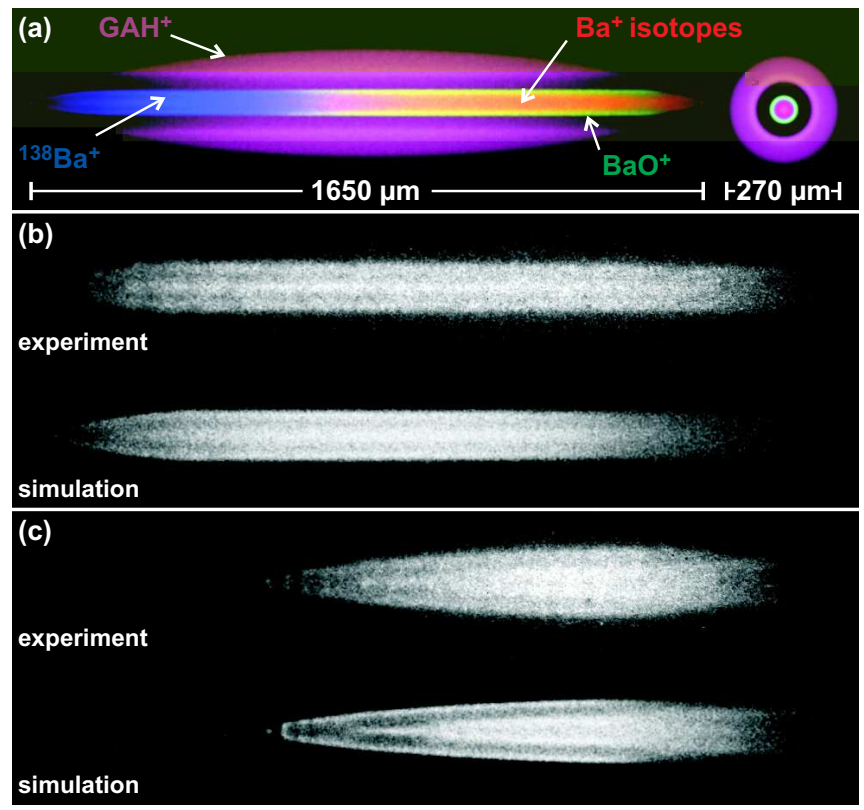

Figure 2. Experimental and simulated CCD images of a $\mathrm{Ba}^{+} / \mathrm{GAH}^{+}$ion crystal. (a) Simulated images in radial (left) and axial view (right), as they would appear if all ions would fluoresce. The cooling lasers propagate to the left and separate the ${ }^{138} \mathrm{Ba}^{+}$ions (blue) and the barium isotopes (red) due to the light pressure force. The $\mathrm{GAH}^{+}$ions (pink) form a sheath around the barium ion subensemble. The $\mathrm{BaO}^{+}$ions (green) are impurities which in this case were not completely removed. (b) Experimental image of the $\mathrm{Ba}^{+} / \mathrm{GAH}^{+}$ion crystal and its simulation, showing only the fluorescing ${ }^{138} \mathrm{Ba}^{+}$ ions. (c) Crystal after removal of the $\mathrm{GAH}^{+}$ions. Here, the barium ion subensemble is no longer deformed nor heated by the $\mathrm{GAH}^{+}$ions.

and $165 \pm 10$ barium isotopest that arrange on the right side of the crystal due to the light pressure force of the cooling lasers propagating to the left and only acting on the laser-cooled species. An additional structural detail in this discussed case is the slight radial constriction on the right side of the barium ion crystal (see figure 2 (c)). This can be ascribed to $\mathrm{BaO}^{+}$or $\mathrm{BaOH}^{+}$ions (in this case $150 \pm 10$ ions) that are generated by photo-induced reactions between ${ }^{138} \mathrm{Ba}^{+}$ions in the $6^{2} \mathrm{P}_{1 / 2}$ excited state and neutral $\mathrm{CO}_{2}, \mathrm{O}_{2}$ or $\mathrm{H}_{2} \mathrm{O}$ molecules from residual gas [23], which was present at an unintendedly high partial pressure in this case. However, under normal operating vacuum conditions $\left(p<10^{-9}\right.$ mbar $)$ the reaction rates are low and the loss of ${ }^{138} \mathrm{Ba}^{+}$is negligible.

From the sharpness and structural details of the barium ion crystals the translational temperatures $T_{s e c, i}=\frac{2}{3}\left\langle E_{i}\right\rangle / k_{B}$ of the ions can be deduced using MD simulations, where $\left\langle E_{i}\right\rangle$ is the time- and subensemble-averaged secular kinetic energy

$\ddagger$ Natural barium consists of seven stable isotopes, the most abundant is ${ }^{138} \mathrm{Ba}$ with a proportion of $72 \%$. In our simulations the other six isotopes are treated as a single species with a weighted mass of $136 \mathrm{amu}$. However, in experimentally observed ion crystals the isotope proportion can be significantly larger than $28 \%$ due to a loss of electronically excited ${ }^{138} \mathrm{Ba}^{+}$ions by laser assisted chemical reactions with residual gas $\mathrm{O}_{2}$ and $\mathrm{CO}_{2}$ molecules that are energetically not possible with the ground state isotope ions [23]. 

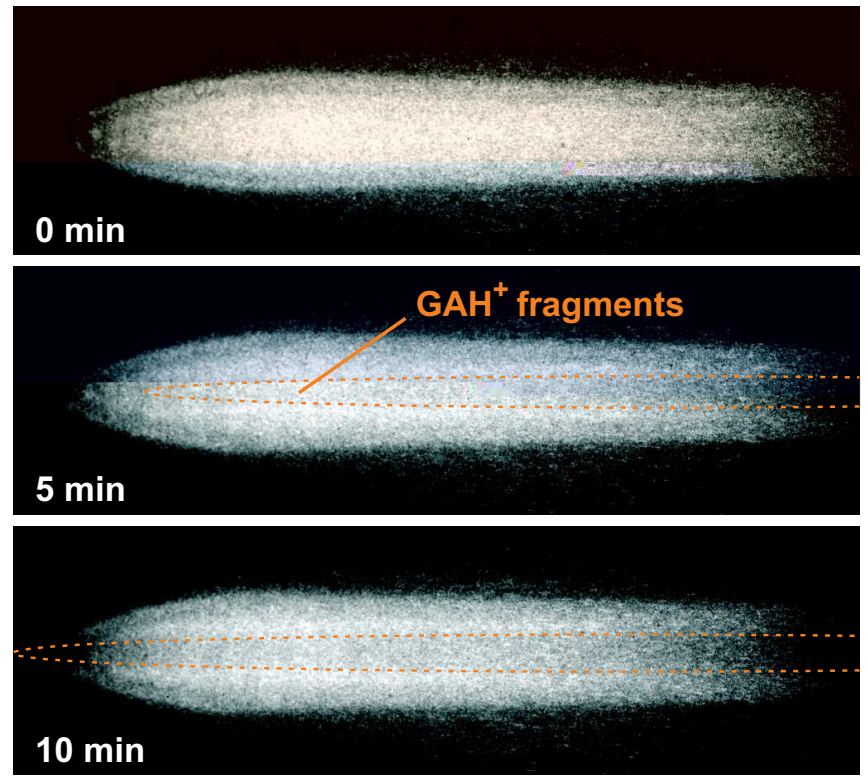

Figure 3. Observation of $\mathrm{GAH}^{+}$photodestruction. An ion crystal consisting of lasercooled ${ }^{138} \mathrm{Ba}^{+}$ions (visible in the CCD image), barium isotopes, $\mathrm{BaO}^{+}$and $\mathrm{GAH}^{+}$ (all three not visible) is continuously exposed to $266 \mathrm{~nm}$ laser light of low intensity. The $\mathrm{GAH}^{+}$ions dissociate within minutes, their fragments accumulate along the trap axis forming a dark core (dashed lines) inside the barium ion subensemble.

per ion of the species $i$ [21]. For the fluorescing ${ }^{138} \mathrm{Ba}^{+}$ions the temperatures can be obtained by a direct comparison of experimental and simulated CCD images, whereas for the sympathetically cooled invisible ions the temperatures can be derived indirectly via the temperature increase of the barium ions due to the sympathetic interaction. As the laser cooling efficiency varies from case to case depending on numerous factors, we consider a realistic range of laser-cooling rates in the simulations in order to obtain the most probable translational temperatures together with a lower and an upper limit. In the discussed case, the $\mathrm{GAH}^{+}$ions had a temperature of $134_{-24}^{+8} \mathrm{mK}$, the $\mathrm{Ba}^{+}$ions 74 $\mathrm{mK}$, the barium isotope ions $122_{-20}^{+5} \mathrm{mK}$ and the $\mathrm{BaO}^{+}$ions $119_{-19}^{+5} \mathrm{mK}$. We expect that $\mathrm{GAH}^{+}$temperatures below $100 \mathrm{mK}$ could be achieved by reducing the ratio of the numbers of $\mathrm{GAH}^{+}$and $\mathrm{Ba}^{+}$ions and the total number of ions, operating at lower residual gas pressures or under improved laser-cooling conditions.

\section{Photodestruction of $\mathrm{GAH}^{+}$}

In the experiment we observe that a $\mathrm{Ba}^{+} / \mathrm{GAH}^{+}$ion crystal exposed to $266 \mathrm{~nm} \mathrm{cw}$ laser light changes its shape due to the destruction of the $\mathrm{GAH}^{+}$ions as shown in figure 3. The produced fragments have lower mass-to-charge ratios than the barium ions and therefore accumulate closer to the trap axis forming a dark core in the barium ion subensemble, which grows during the dissociation process. The fragments' mass-to-charge ratios range between 40 and $82 \mathrm{Da}$ as determined by a mass-selective extraction of the ions from the trap (see section 3.2). A destruction of $\mathrm{GAH}^{+}$by the barium cooling lasers (493 
and $650 \mathrm{~nm}$ ) alone has not been observed. Here, we show two methods to measure quantitatively the decay of the $\mathrm{GAH}^{+}$parent ion number. In the "excitation method" the decay is detected during one photodestruction process via a repeated excitation of the parent ions that produces signals proportional to the number of parent ions. In the "extraction method" the composition of a set of initially similar $\mathrm{Ba}^{+} / \mathrm{GAH}^{+}$ion crystals is analyzed via extractions of the ions from the trap after different times of $\mathrm{UV}$ exposition. The analysis of the $\mathrm{GAH}^{+}$parent ion number decay relies on the rate equation model derived in section 3.3. In this model, the parent ion number $N(t)$ after a UV exposure time $t$ is given by

$$
N(t)=N(0) \exp (-\gamma t)
$$

with the photodestruction rate $\gamma$. Note that this rate is not a photodissociation rate in the sense of an inverse lifetime of an excited molecule against dissociation. Rather, it is determined by the absorption rate times the sum of fractional probabilities for various dissociation pathways, such as unimolecular, statistical, and nonstatistical dissociation. The various pathways cannot be specified further due to the principle of our measurements.

\subsection{Excitation method}

In the harmonic, effective electric potential of a linear quadrupole trap [24], ions can oscillate radially with a mass-to-charge ratio specific resonance frequency

$$
\omega_{r}=\frac{\Omega}{2} \sqrt{\frac{q^{2}}{2}+a}
$$

with the Mathieu stability parameters

$$
q=\frac{2 Q U_{\mathrm{RF}}}{m r_{0}^{2} \Omega^{2}}
$$

and

$$
a=\frac{-4 \kappa Q U_{\mathrm{EC}}}{m \Omega^{2}} .
$$

Typical trap parameters in our case are $U_{\mathrm{RF}}=200-500 \mathrm{~V}$ for the trap RF amplitude, $U_{\mathrm{EC}}=5-7 \mathrm{~V}$ for the DC potential difference between the end and middle segments of the trap electrode rods (see figure 11). Constant parameters are the RF frequency $\Omega=2 \pi \cdot 2.5 \mathrm{MHz}$, the axis-to-electrode-surface distance $r_{0}=4.36 \mathrm{~mm}$, and the geometrical factor $\kappa=1500 \mathrm{~m}^{-2}$.

These oscillations can be excited by AC voltages applied to the trap electrodes or an additional excitation electrode (see figure 1). When the excitation frequency coincides with the resonance frequency of an ion species, its strong large-amplitude response heats up all trapped ions due to their mutual Coulomb interaction. As a consequence, the barium ions' fluorescence drops, leading to dips in the recorded fluorescence signal as a function of the AC frequency [25, 26]. The depth of theses dips is approximately 
Measurement of small photodestruction rates of cold, charged biomolecules in an ion trap 8

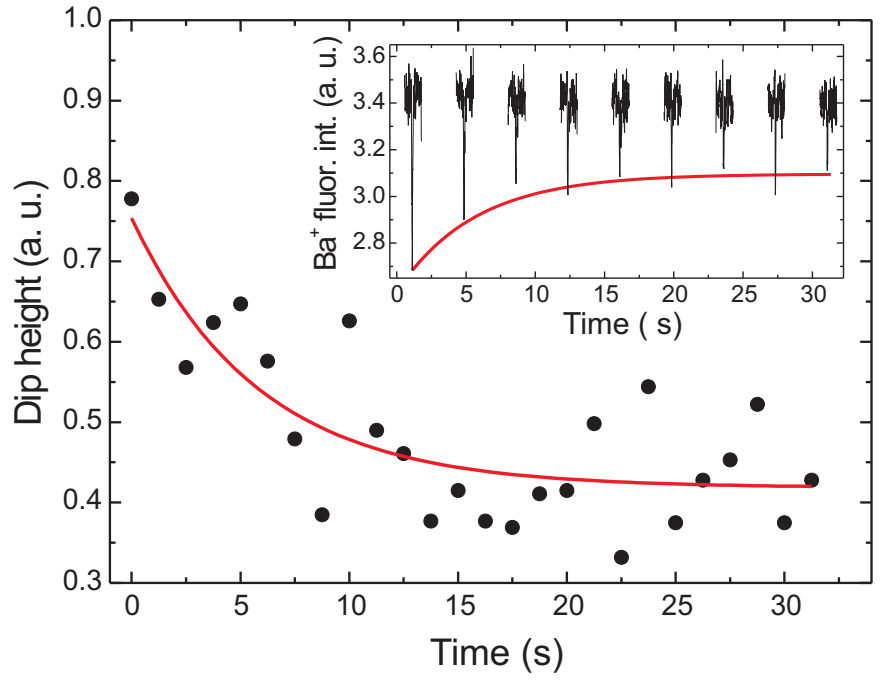

Figure 4. Photodestruction of $\mathrm{GAH}^{+}$measured with the excitation method. The inset shows repeated radial excitation scans over the $\mathrm{GAH}^{+}$resonance with their height decreasing during the photodissociation of the $\mathrm{GAH}^{+}$parent ions by a $266 \mathrm{~nm}$ laser at $12 \mathrm{~mW} / \mathrm{cm}^{2}$. Due to the occurrence of a new dip in the scanned frequency range, the dips do not vanish completely. The main plot shows the dip heights of the complete measurement with an exponential fit. The average of six such measurements at this intensity yields a rate of $\gamma=(0.15 \pm 0.02) \mathrm{s}^{-1}$.

proportional to the number of excited ions [27]. This was confirmed by comparison of the results of the two methods presented here (see section 3.3).

Thus, the decay of the number of a sympathetically cooled ion species can be followed by recording the barium ions' fluorescence during repeated frequency scans over the species' resonance. The result of such a measurement is shown in figure 4 for the photodestruction of $\mathrm{GAH}^{+}$with $266 \mathrm{~nm}$ laser light at an intensity of $12 \mathrm{~mW} / \mathrm{cm}^{2}$. The radial resonance of $\mathrm{GAH}^{+}$was repetitively excited via the external excitation electrode by frequency scans from 35 to $15 \mathrm{kHz}$ with a duration of $0.2 \mathrm{~s}$ and a repetition rate of $0.8 \mathrm{~Hz}$. For clarity, the inset of figure 4 shows only every third resonance dip of this destruction process, while the dip heights of the complete measurement are shown in the main plot. An exponential fit to the decay of the $\mathrm{GAH}^{+}$resonance dip height, which equals the decay of the $\mathrm{GAH}^{+}$parent ion number, yields the destruction rate $\gamma$. The average of six such measurements at this intensity of $12 \mathrm{~mW} / \mathrm{cm}^{2}$ yields a rate of $\gamma=(0.15 \pm 0.02) \mathrm{s}^{-1}$. Several measurements with different dissociation laser intensities have been performed both with this and the extraction method; the results are discussed later and shown in figure 7 .

Unfortunately, for this test molecule $\mathrm{GAH}^{+}$and probably for other molecules with similar masses ( $471 \pm 50 \mathrm{Da})$ this method is ambiguous. With the parent ion number decreasing to zero, their resonance dips should vanish completely during the dissociation process. However, we cannot observe this for $\mathrm{GAH}^{+}$(see figure 44). Here, during the dissociation a new resonance arises close to the parent ion resonance which cannot be resolved and just slightly broadens the observed parent ion dip. We can explain this 
new resonance as being due to the generated fragment ions. Their mass-to-charge ratios of 40 to 82 Da lead not only to radial resonances between 260 to $130 \mathrm{kHz}$ as expected from equation 4 with $U_{\mathrm{RF}}=291 \mathrm{~V}$ as applied in the case of figure 4, but also to an additional resonance which coincidentally agrees with that of the parent ions $(\approx 22 \mathrm{kHz}$ in this case). This can be explained by a motional coupling of all trapped ions [26]. Even so, an evaluation of the rates of the $\mathrm{GAH}^{+}$dissociation process measured with this method was possible (as in figure 44) and lead to results that agree well with those measured with the extraction method (see figure 7 , open circles). For other parent ion masses sufficiently different from that of $\mathrm{GAH}^{+}$, this problem will not occur, as their resonances can be resolved from those arising from their fragments. Generally speaking, the mass resolution of radial excitation depends on the numbers and mass-to-charge ratios of the trapped ion species of a specific ion ensemble and is limited by motional coupling effects. The mass resolution is in the order of about $10 \%$ but coupling effects can lead to ambiguous results as mentioned above.

\subsection{Extraction method}

In the extraction method we make use of a mass-to-charge ratio selective extraction of the ions from the trap. When decreasing the trap RF amplitude $U_{\mathrm{RF}}$ the Mathieu stability parameter $q$ (see equation 5 ) is reduced and the ions escape from the trap at a mass-to-charge ratio dependent amplitude

$$
U_{\mathrm{RF}}^{\mathrm{ex}}=\alpha \cdot r_{0}^{2} \Omega \sqrt{2 \kappa U_{\mathrm{EC}} \frac{m}{Q}} .
$$

This expression with $\alpha=1$ follows from the Mathieu stability diagram (e. g. shown in [24]) with regard to the decrease of $q$ below stability\&. In our setup, an extraction plate (see figure 1) $17 \mathrm{~mm}$ below the trap center at a potential of $-1000 \mathrm{~V}$ referred to ground draws the escaped ions to an ion detector that registers these ions during such a controlled reduction of the RF amplitude, leading to an ion extraction mass spectrum as shown in figure 5. The overall ion capture efficiency is between 10 and $20 \%$ depending mainly on the applied detector electronics settings. During the experiments the efficiency is constant and almost equal for the various singly charged species. This was determined by comparing ion numbers detected by the counter with the numbers in the Coulomb crystals determined by molecular dynamics simulations [21]. The numerical factor $\alpha>1$ arises from the attracting effect of the extraction plate that causes the ions to escape the trap earlier during the RF amplitude reduction, i. e. at higher amplitudes. Its value slightly varies depending on ion numbers and temperatures and is approximately 2.4 in our setup. Using the barium peak in an ion extraction mass spectrum for a precise calibration of $\alpha$, we find that we can then verify the mass of $\mathrm{GAH}^{+}$and determine the mass-to-charge ratio range of its fragments to $40-82$ Da (see figure 5).

$\S$ This holds when the edge of the stability range is approximated by $q=-\frac{1}{2} a^{2}$ which is adequate for the range of $q$ considered here. 
Measurement of small photodestruction rates of cold, charged biomolecules in an ion trap 10

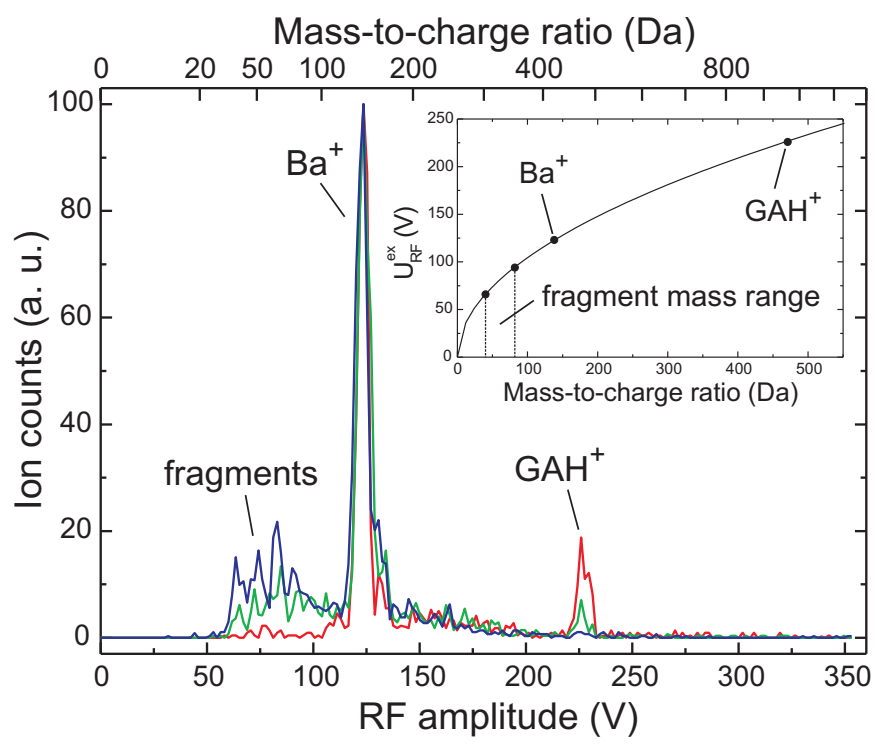

Figure 5. Ion extraction mass spectra of $\mathrm{Ba}^{+} / \mathrm{GAH}^{+}$crystals at different stages of $\mathrm{GAH}^{+}$dissociation. The red curve is the extraction spectrum of an intact $\mathrm{Ba}^{+} / \mathrm{GAH}^{+}$ crystal showing only the two species. The green curve shows the extraction of an identically prepared crystal after a $1.2 \mathrm{~s}$ exposition to $266 \mathrm{~nm}$ laser light at $55 \mathrm{~mW} / \mathrm{cm}^{2}$. Here, the number of $\mathrm{GAH}^{+}$ions is reduced and fragments appear. After $2.4 \mathrm{~s}$ (blue curve) all $\mathrm{GAH}^{+}$ions are dissociated and the number of fragments reaches a final maximum. The inset curve shows the theoretical dependency of the extraction RF amplitude $U_{\mathrm{RF}}^{\mathrm{ex}}$ on the mass-to-charge ratio, calibrated with the known mass of barium. The $\mathrm{GAH}^{+}$data point indicates that indeed intact $\mathrm{GAH}^{+}$molecules have been stored.

This mass identification technique is destructive, but it has several advantages compared to the non-destructive radial excitation. It unambiguously distinguishes species according to their mass-to-charge ratio reaching a resolution $<20$ Da below $\sim 500 \mathrm{Da}$ for cold ensembles. Furthermore, it is also applicable to uncooled ensembles, however with a lower resolution, and it is fast because an extraction only takes a few seconds without any preparation.

In order to measure photodestruction rates with this technique, ion crystals prepared under identical conditions containing the desired molecular ions are exposed to the dissociation laser for different, sequentially increased times. The resulting ion crystals in different stages of the destruction process are analyzed via ion extraction. Thus, the decay of the parent ion number is fully described by such a set of ion extraction spectra. Figure 5 shows ion extraction spectra of identically prepared $\mathrm{Ba}^{+} / \mathrm{GAH}^{+}$ crystals in different stages of the $\mathrm{GAH}^{+}$dissociation by $266 \mathrm{~nm}$ laser light of $55 \mathrm{~mW} / \mathrm{cm}^{2}$ intensity. The red curve is the extraction spectrum of an intact $\mathrm{Ba}^{+} / \mathrm{GAH}^{+}$crystal acquired directly after preparation showing only the two species. The green curve was acquired after an UV exposition of $1.2 \mathrm{~s}$ and shows a reduced number of $\mathrm{GAH}^{+}$ions and the appearance of fragments. After $2.4 \mathrm{~s}$ all $\mathrm{GAH}^{+}$ions are dissociated and the number of fragments reaches a final maximum as shown in the blue curve. 
A parent ion decay curve is obtained from such a set of ion extraction spectra by adding up the ion counts of the parent ion peak in each extraction spectrum. However, although all starting ion crystals of a dissociation run are prepared under identical conditions, there are deviations in the numbers of loaded molecular ions with a standard deviation of up to $28 \%$. This is due to the irregular molecular ion flux of the ESI ion source that shows as similar standard deviation of $25 \%$, a typical value for ESI sources [28]. Therefore, several complete dissociation runs are measured for the same spectroscopy condition (laser intensities, wavelengths) in order to minimize this nonsystematic error. In the discussed case (see above and figure 5) four $\mathrm{GAH}^{+}$dissociation runs have been acquired leading to the decay curves shown in the inset of figure 6. The main plot shows the averaged data points of these curves and an exponential fit that yields a rate $\gamma=(1.00 \pm 0.13) \mathrm{s}^{-1}$ for this photodestruction process.

\subsection{Discussion}

The two presented methods are based on fundamentally different principles and therefore show diverse advantages and difficulties. The most striking difference is the time it takes to measure a destruction rate for a given spectroscopy condition. Under best experimental conditions, such a measurement takes a few minutes with the excitation method, whereas it takes at least one hour with the extraction method. This is because in the latter case dozens of ion crystals need to be prepared and destructed to achieve lower relative uncertainties, compared to only one crystal in the first case.

However, this speed difference is often compensated by the higher experimental

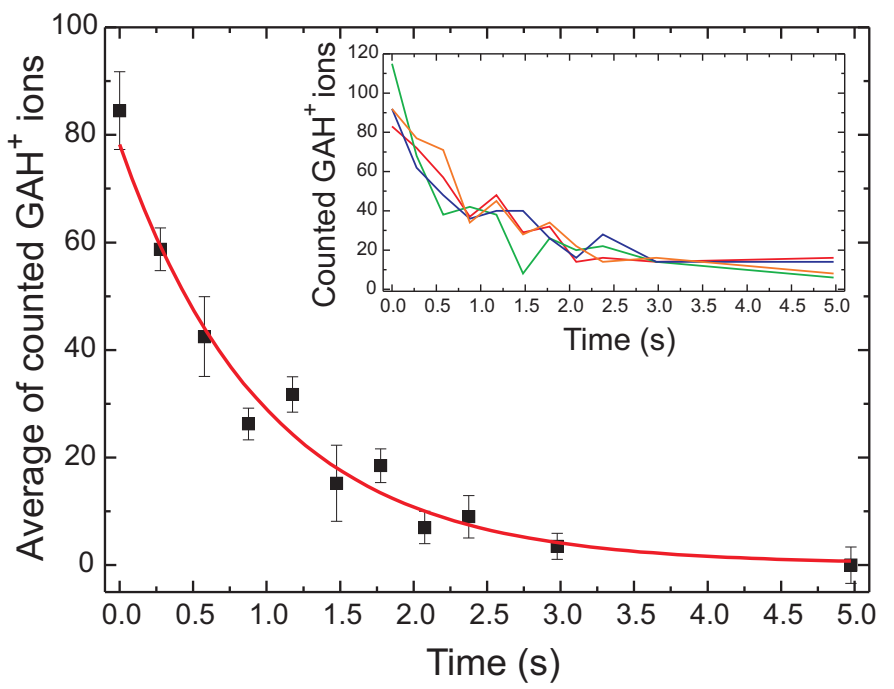

Figure 6. Photodissociation of $\mathrm{GAH}^{+}$measured with the extraction method for an exposition with $266 \mathrm{~nm}$ laser light at $55 \mathrm{~mW} / \mathrm{cm}^{2}$. The inset shows the decay of the $\mathrm{GAH}^{+}$parent ion number of four sets of 11 ensembles, where the crystals of each set were UV-exposed consecutively for increasing durations. The main plot shows the decay of the $\mathrm{GAH}^{+}$numbers, averaged over the four sets shown in the inset. The exponential fit yields a photodestruction rate $\gamma=(1.00 \pm 0.13) \mathrm{s}^{-1}$. 
Measurement of small photodestruction rates of cold, charged biomolecules in an ion trap 12

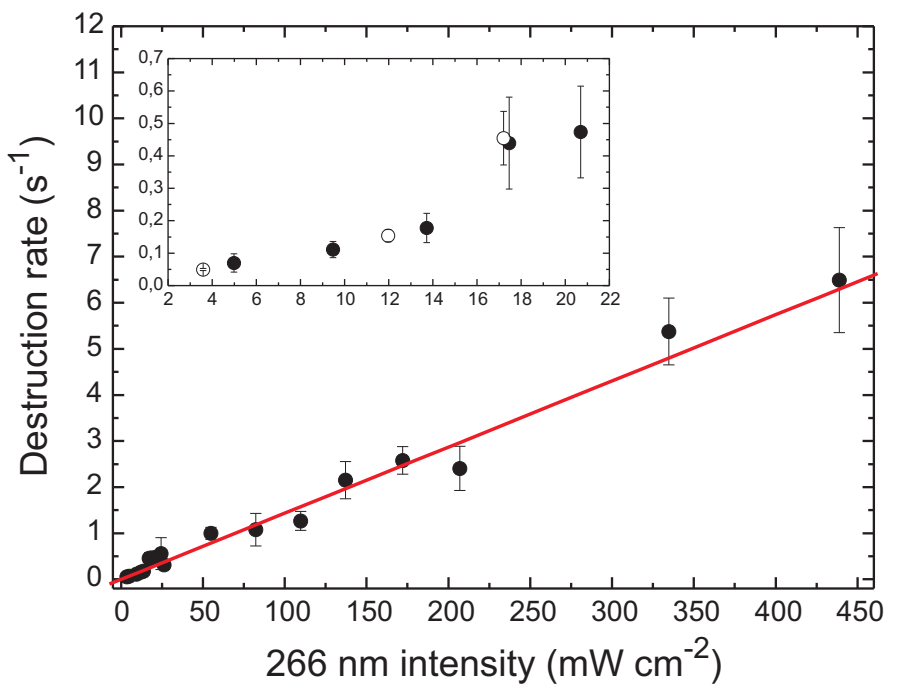

Figure 7. Destruction of translationally cold $\mathrm{GAH}^{+}$at different laser intensities. The destruction rates shows a linear dependence on the $266 \mathrm{~nm}$ dissociation laser intensity in the range of intensities covered (the red line is a linear fit). The rates measured with the excitation method (open circles, inset) agree well with those measured with the extraction method (filled circles).

effort of the excitation method. Here, it is a complex task to produce ion crystals with a certain required critical ratio of numbers of laser-cooled atomic ions and sympathetically cooled molecular ions. Two conditions need to be balanced - the signal-to-noise ratio of the resonance dips needs to be sufficiently high and the ion ensembles need to stay in the crystal state during the whole dissociation process. The first condition, a high signal-to-noise ratio, can be easily achieved with a higher number of molecular ions that is only limited by the finite sympathetic cooling power of the laser-cooled ions. However, the maintenance of the crystal state is more critical, because every resonant excitation of the ions implies a disturbance. When the number of molecular ions is too large, the ion crystals melt before the dissociation process is finished, as they are perturbed by the produced fragment ions due to their stronger sympathetic interaction with the lasercooled ions. In addition, with higher numbers of molecular ions and their fragments, the ion crystals are more sensitive, so that slightly stronger or more frequent excitations can easily melt the crystals.

Thus, the preparation and the handling of barium/molecule ion crystals for the application of the excitation technique requires a precise and critical control of the loading and excitation parameters. In comparison, the extraction method is less sensitive but more straightforward, as it does without the crucial excitation. Here, the only condition to be fulfilled, is to prepare ion crystals with a sufficient number of molecular ions that produces a distinct peak in the ion extraction spectra. We expect that this method could be automated to a large extent for the application in spectroscopic studies.

To demonstrate the practicability of our methods, we have investigated the photodestruction of $\mathrm{GAH}^{+}$with a cw $266 \mathrm{~nm}$ laser at low intensities ranging from 3 to 
$440 \mathrm{~mW} / \mathrm{cm}^{2}$. Figure 7 shows the photodestruction rates obtained with the extraction method (filled circles) and the excitation method (open circles, inset). The measured rates range from 0.05 to $6.5 \mathrm{~s}^{-1}$ and linearly depend on the dissociation laser intensity. Significantly lower rates should in principle be observable due to the long-term stable storage of the molecular ions of up to hours. Measurements of rates higher than $0.5 \mathrm{~s}^{-1}$ are not possible with the excitation method as the excitation repetition rate is limited to $\lesssim 2 \mathrm{~Hz}$. With higher repetition rates the crystals cannot cool down sufficiently after excitation to maintain crystallization. In contrast, for the extraction method the upper limit of measurable rates is only limited by the shutting time of the dissociation lasers which is in the range of milliseconds using mechanical shutters or nanoseconds for electro-optical switches.

With our apparatus we cannot and do not intend to give insight into the precise nature of the destruction process of $\mathrm{GAH}^{+}$or other molecular species. Our mass resolution is too low to distinguish all generated fragment ion species. In addition, the further dissociation of fragments due to the comparatively long UV exposure times in the measurements presented here complicates any kind of interpretation. Various pathways for the unimolecular dissociation are possible like loss of neutral or charged groups or cleavage of the molecules at weak bonds [9]. Generally, gas-phase biomolecules of sizes comparable to that of $\mathrm{GAH}^{+}$can dissociate after the absorption of a single UV photon of $266 \mathrm{~nm}$ [9, 29, 30, which we well confirmed by the linear intensity dependence of the measured destruction rates of $\mathrm{GAH}^{+}$. After absorption, such molecules either dissociate within less than microseconds from an electronically excited state (nonstatistical dissociation) or on a timescale of milliseconds after redistribution of the internal energy to vibrational modes (statistical dissociation). Especially for protonated molecules of sizes comparable to that of $\mathrm{GAH}^{+}$, the "immediate" nonstatistical dissociation is the dominant process [30]. Based on this assumption the destruction process can be described by a simple rate equation model as motivated in [18] given by

$$
\begin{aligned}
& \frac{d N_{e}(t)}{d t}=N_{g}(t) \cdot R-N_{e}(t) \cdot(R+\Gamma+k) \\
& \frac{d N_{g}(t)}{d t}=-N_{g}(t) \cdot R+N_{e}(t) \cdot(R+\Gamma)
\end{aligned}
$$

Here, $\mathrm{GAH}^{+}$ions in the electronic ground state with an ion number $N_{g}(t)$ absorb laser photons with the absorption rate $R=\sigma_{a b s} I / h \nu$, where $\sigma_{a b s}$ is the absorption cross section of $\mathrm{GAH}^{+}$and $I$ the intensity of the dissociation laser at the frequency $\nu=c /(266 \mathrm{~nm}) . \quad N_{e}(t)$ is the ion number in the electronically excited state. Its decay is characterized by the fluorescence rate $\Gamma$ and its photodissociation channel by the unimolecular dissociation rate $k$. The solution for the total parent ion number $N(t)=N_{g}(t)+N_{e}(t)$ in the relevant case $R \ll k, \Gamma$ at the low intensities $I$ used in our measurements is given by

$$
N(t)=N(0) \cdot \exp (-\gamma t)
$$


Measurement of small photodestruction rates of cold, charged biomolecules in an ion trap 14

with the photodestruction rate

$$
\gamma=\frac{k R}{k+\Gamma} .
$$

With the relation $\gamma=\sigma \cdot I / h \nu$ valid for single-photon processes, we define a photodestruction cross section

$$
\sigma_{p d}=\sigma_{a b s} \frac{k}{k+\Gamma} .
$$

A linear fit to the data of our measurement shown in figure 7 yields a photodestruction cross section at $266 \mathrm{~nm}$ of translationally cold, trapped $\mathrm{GAH}^{+}$ions of $\sigma_{p d}=(1.1 \pm 0.1) \cdot 10^{-17} \mathrm{~cm}^{2}$.

Only few quantitative measurements on UV photodestruction cross sections of trapped molecular ions have been performed. Similar measurements on trapped, but warm $\mathrm{CH}_{4}^{+}$[17] yielded smaller photodestruction cross sections of the order of $1 \cdot 10^{-19}$ $\mathrm{cm}^{2}$ for visible wavelengths, with a tendency to increase with decreasing wavelength. In our model, for high photodissociation rates $k \gg \Gamma$, the photodestruction cross section $\sigma_{p d}$ would equal the absorption cross section $\sigma_{a b s}$, while in any other case it would be less. There are, to our knowledge, no quantitative measurements on the UV absorbtion cross section of $\mathrm{GAH}^{+}$so that we can only compare the lower limit $\left(\sigma_{a b s}\right)_{\min }=\sigma_{p d}$ with absorption cross sections of other gas-phase organic molecules. For example, a gas-phase amide $\left(\mathrm{C}_{4} \mathrm{H}_{9} \mathrm{NO}\right)$ shows an absorption cross section at $266 \mathrm{~nm}$ of $\sigma_{\text {abs }}(266 \mathrm{~nm}) \approx 5 \cdot 10^{-19}$ $\mathrm{cm}^{2}\left[31\right.$ and neopentylperoxy radical molecules $\left(\mathrm{C}_{5} \mathrm{H}_{11} \mathrm{O}_{2}\right) \sigma_{a b s}(266 \mathrm{~nm}) \approx 4 \cdot 10^{-18} \mathrm{~cm}^{2}$ [32, that are both lower than that of $\mathrm{GAH}^{+}$. Measurements on fullerenes $\left(\mathrm{C}_{60}\right)$ at the same wavelength gave $\sigma_{a b s}(266 \mathrm{~nm}) \approx 4 \cdot 10^{-16} \mathrm{~cm}^{2}[33]$.

\section{Conclusion}

In this work, we have shown two methods for the measurement of photodestruction rates of translationally cold, trapped molecular ions. A particular advantage of our technique are the long storage times of many minutes and in principle of up to hours in the welldefined and nearly collisionless environment of an ion trap in an ultrahigh vacuum. This allows for the study of slow destruction processes such as the photodissociation of large biomolecules. For trapped and translationally cooled, singly protonated ions of the organic compound glycyrrhetinic acid a photodestruction cross section at 266 $\mathrm{nm}$ of $(1.1 \pm 0.1) \cdot 10^{-17} \mathrm{~cm}^{2}$ has been determined. In future, the methods could be applied for a systematic acquisition of highly resolved photodissociation spectra using low intensity, tunable cw lasers with narrow linewidths. In order to reduce the spectral congestion due to the presence of numerous conformers, a cooling of the internal degrees of freedom would be highly advantageous and could be implemented by radiative cooling in a cryogenic environment [16]. 
Measurement of small photodestruction rates of cold, charged biomolecules in an ion trap 15

\section{Acknowledgments}

We thank R. Edrada-Ebel and R. Weinkauf for helpful discussions. DO acknowledges support from the Studienstiftung des deutschen Volkes and CBZ from the Deutscher Akademischer Austauschdienst (DAAD). This work was supported by the German Science Foundation.

\section{References}

[1] Correia C F, Balaj P O, Scuderi D, Maitre P and Ohanessian G 2008 Vibrational Signatures of Protonated, Phosphorylated Amino Acids in the Gas Phase J. Am. Chem. Soc. 130 3359-70

[2] Stearns J A, Guidi M, Boyarkin O V and Rizzo T R 2007 Conformation-specific infrared and ultraviolet spectroscopy of tyrosine-based protonated dipeptides J. Chem. Phys. 127154322

[3] Dienes T et al 1996 Fourier Transform Mass Spectrometry - Advancing Years Mass Spectrom. Rev. 15 163-211

[4] Nolting D, Schultz T, Hertel I V and Weinkauf R 2006 Excited state dynamics and fragmentation channels of the protonated dipeptide $\mathrm{H}_{2} \mathrm{~N}-\mathrm{Leu}-\mathrm{Trp}-\mathrm{COOH}$ Phys. Chem. Chem. Phys. 8 5247-54

[5] Price W D, Schnier P D and Williams E R 1996 Tandem Mass Spectrometry of Large Biomolecule Ions by Blackbody Infrared Radiative Dissociation Anal. Chem. 68 859-66

[6] Griffin L L and McAdoo D J 1993 The Effect of Ion Size on Rate of Dissociation: RRKM Calculations on Model Large Polypeptide Ions J. Am. Soc. Mass Spectrom. 4 11-15

[7] Schlag E W and Levine R D 1989 On the unimolecular dissociation of large molecules Chem. Phys. Lett. 163 523-30

[8] Dunbar R C 2000 Photodissociation of trapped ions Int. J. Mass Spectrom. 200 571-89

[9] Worm E S et al 2007 Photodissociation of dinucleotide ions in a storage ring Phys. Rev. A 75 042709

[10] Støchkel K et al 2008 A new technique for time-resolved daughter ion mass spectrometry on the microsecond to millisecond time scale using an electrostatic ion storage ring Rev. Sci. Instrum. 79023107

[11] Højbjerre K et al 2008 Consecutive photodissociation of a single complex molecular ion Phys. Rev. A 77 030702(R)

[12] Stearns J A, Boyarkin O V and Rizzo T R 2007 Spectroscopic Signatures of Gas-Phase Helices: Ac-Phe-(Ala) $)_{5}-\mathrm{Lys}_{-} \mathrm{H}^{+}$and Ac-Phe-(Ala) ${ }_{10}-\mathrm{Lys}_{-} \mathrm{H}^{+}$J. Am. Chem. Soc. 129 13820-1

[13] Koelemeij J C J, Roth B, Wicht A, Ernsting I and Schiller S 2007 Vibrational Spectroscopy of $\mathrm{HD}^{+}$with 2-ppb Accuracy Phys. Rev. Lett. 98173002

[14] Ostendorf A et al 2006 Sympathetic Cooling of Complex Molecular Ions to Millikelvin Temperatures Phys. Rev. Lett. 97 243005; Ostendorf A et al 2008 Erratum: Sympathetic Cooling of Complex Molecular Ions to Millikelvin Temperatures Phys. Rev. Lett. 100 019904(E)

[15] Offenberg D, Zhang C B, Wellers C, Roth B and Schiller S 2008 Translational cooling and storage of protonated proteins in an ion trap at sub-Kelvin temperatures Phys. Rev. A 78 061401(R)

[16] Berkeland D J, Miller J D, Bergquist J C, Itano W M and Wineland D J 1998 Laser-Cooled Mercury Ion Frequency Standard Phys. Rev. Lett. 80 2089-92

[17] Ensberg E S and Jefferts K B 1975 The visible photodissociation spectrum of ionized methane Astrophys. J. 195 L89-91

[18] Khoury J T, Rodriguez-Cruz S E and Parks J H 2002 Pulsed Fluorescence Measurements of Trapped Molecular Ions with Zero Background Detection J. Am. Soc. Mass. Spectrom. 13 696708

[19] Fenn J B, Mann M, Meng C K, Wong S F and Whitehouse C M 1989 Electrospray Ionization for Mass Spectrometry of Large Biomolecules Science 246 64-71 
[20] Roth B, Ostendorf A, Wenz H and Schiller S 2005 Production of large molecular ion crystals via sympathetic cooling by laser-cooled $\mathrm{Ba}^{+} J$. Phys. B 38 3673-85

[21] Zhang C B, Offenberg D, Roth B, Wilson M A and Schiller S 2007 Molecular-dynamics simulations of cold single-species and multispecies ion ensembles in a linear Paul trap Phys. Rev. A 76012719

[22] Wineland D J 1987 Proceedings of the cooling, condensation and storage of hydrogen cluster ions workshop (Menlo Park) p 181 ed J T Bahns

[23] Roth B, Offenberg D, Zhang C B and Schiller S 2008 Chemical reactions between cold trapped $\mathrm{Ba}^{+}$ions and neutral molecules in the gas phase, Phys. Rev. A $\mathbf{7 8} 042709$

[24] Drewsen M and Brøner A 2000 Harmonic linear Paul trap: Stability diagram and effective potentials Phys. Rev. A 62045401

[25] Baba T and Waki I 2002 Spectral shape of in situ mass spectra of sympathetically cooled molecular ions J. Appl. Phys. 92 4109-16

[26] Roth B, Blythe P and Schiller S 2007 Motional resonance coupling in cold multispecies Coulomb crystals Phys. Rev. A $\mathbf{7 5} 023402$

[27] Roth B, Koelemeij J C J, Daerr H and Schiller S 2006 Rovibrational spectroscopy of trapped molecular hydrogen ions at millikelvin temperatures Phys. Rev. A 74 040501(R)

[28] Rizzo T R 2008, private communication

[29] Liu B, Hvelplund P, Nielsen S B and Tomita S 2006 Photodissociation of singly charged oligonucleotide cations: Arrhenius parameters and identification of nonstatistical processes Phys. Rev. A 74052704

[30] Nielsen S B 2004 Gas-Phase Studies of Chromophore Molecules in an Electrostatic Storage Ring Phys. Scr. T110 332-5

[31] Chakir A, Solignac G, Mellouki A and Daumont D 2005 Gas phase UV absorption cross-sections for a series of amides Chem. Phys. Lett. 404 74-8

[32] Dagaut P and Kurylo M J 1990 A Flash Photolysis Investigation of the Gas Phase UV Absorption Spectrum and Self-Reaction Kinetics of the Neopentylperoxy Radical Int. J. Chem. Kin. 22 1177-87

[33] Smith A L 1996 Comparison of the ultraviolet absorption cross section of $\mathrm{C}_{60}$ buckminsterfullerene in the gas phase and in hexane solution J. Phys. B 29 4975-80 